\title{
The Existence and Uniqueness of a New Boundary Value Problem (Type of Problem "E") for Linear System Equations of the Mixed Hyperbolic-Elliptic Type in the Multivariate Dimension with the Changing Time Direction
}

\author{
Mahammad A. Nurmammadov ${ }^{1,2}$ \\ ${ }^{1}$ Department of Mathematics, Azerbaijan Architecture and Building University, 5 A. Sultanova Street, 1073 Baku, Azerbaijan \\ ${ }^{2}$ Department of Mathematics, Azerbaijan Teachers Institute, 104 H. Aliyev Street, 1115 Baku, Azerbaijan \\ Correspondence should be addressed to Mahammad A. Nurmammadov; nurmamedov@mail.ru
}

Received 25 October 2014; Revised 5 January 2015; Accepted 28 January 2015

Academic Editor: Patricia J. Y. Wong

Copyright (c) 2015 Mahammad A. Nurmammadov. This is an open access article distributed under the Creative Commons Attribution License, which permits unrestricted use, distribution, and reproduction in any medium, provided the original work is properly cited.

\begin{abstract}
The existence and uniqueness of the boundary value problem for linear systems equations of the mixed hyperbolic-elliptic type in the multivariate domain with the changing time direction are studied. Applying methods of functional analysis, " $\varepsilon$-regularizing" continuation by the parameter and by means of prior estimates, the existence and uniqueness of generalized and regular solutions of a boundary problem are established in a weighted Sobolev space.
\end{abstract}

Dedicated to Prof. V. N. Vragov on the occasion of his 70th birthday.

\section{Introduction}

Up to the present, various generalizations of mixed and composite type of equations have been investigated by many authors. Interest of investigations of nonclassical equations arises in applications in the field of hydrogas dynamics, modeling of physical processes (see, e.g., [1-6] and the references given therein).

Nonclassical model is defined as the model of mathematical physics, which is represented in the form of the equation or systems of partial differential equations that does not fit into one of the classical types: elliptic, parabolic, or hyperbolic. In particular, nonclassical models are described by equations of mixed type (the Tricomi equation), degenerate equations (the Keldysh equation) or the equations of Sobolev type (or the Barenblatt-Zsolt-Kachina equation), the equation of mixed type with the changing time direction, and forward-backward equations. In [7], a fundamental theory of the solvability of many different boundary value problems for nonclassical equations was presented.

In recent years the attention of many scholars has turned to the study of well-posed boundary value problems for nonclassical equations of mathematical physics, in particular, for forward-backward equations of the parabolic type (as the changing time direction) (see, e.g., $[8,9]$, etc.) and for equations of mixed type with several lines of change of type (see, e.g., [8-12] and the references given therein).

In the theory of boundary value problems for degenerate equations and equations of mixed type, it is a well-known fact that the well-posedness and the class of its correctness essentially depend on the coefficient of the first-order derivative (younger member) of equations (e.g., [13-16] and the references given therein).

In [14] the new called Fichera function was introduced, in order to identify subsets of the boundary of the domain where the boundary value problem for such kind of equations 
is posed, where it is necessary (or not) to specify the boundary condition. A namely, the boundary conditions depends on sign of function Fichera's $\Phi(x)$.

In [13] (see, Chapter 1, pages 191-197, and Chapter 3, pages 239-245) and [15] new boundary conditions (so-called type of problem " $E$ " in which some part of the boundary will be exempt from the boundary conditions) were studied.

In [16-18] various Dirichlet problems which can be formulated for equations of Keldysh type, one of the two main classes of linear elliptic-hyperbolic equations, were investigated. Open boundary conditions (in which data are prescribed on only part of the boundary) and closed boundary conditions (in which data are prescribed on the entire boundary) were both considered. Emphasis is on the formulation of boundary conditions for which solutions can be shown to exist in an appropriate function space.

Boundary value problems for equations of mixed hyperbolic-elliptic type with changing time direction had been studied in detail in [10-12]. Great difficulties come into being in the investigation of systems of degenerate elliptic and hyperbolic equations.

Note that many authors studied hyperbolic-parabolic equations and system equations of degenerating elliptic type, system equations of hyperbolic-elliptic type and system equations of hyperbolic (see, e.g., $[3,5,6,19]$ and the references given therein).

In mathematical modeling, partial differential equations of the mixed type are used together with boundary conditions specifying the solution on the boundary of the domain. In some cases, classical boundary conditions cannot describe process or phenomenon precisely. Therefore, mathematical models of various physical, chemical, biological, or environmental processes often involve nonclassical conditions. Such conditions usually are identified as nonlocal boundary conditions and reflect situations when the data on the domain boundary cannot be measured directly or when the data on the boundary depend on the data inside the domain. In numerical methods for solving these equations, the problem of stability has received a great deal of importance and attention. In particular, a suitable model for analyzing the stability is provided by a proper unconditionally absolutely stable difference scheme with an unbounded operator. The method of operators as a tool for the investigation of the stability of the solution of nonlocal problems for partial differential equations of the mixed type in Hilbert and Banach spaces and of difference schemes for approximate solution of these problems has been systematically developed (see, e.g., [20-22] and the references given therein).

Finally, the problem for the system of equations of hyperbolic-elliptic type, including property of changing time direction, has not been extensively investigated. Therefore in the present paper we will study this problem.

\section{Problem Statement, Notation, and Preliminaries}

Let $G$ be a bounded domain in the Euclidean space $R^{n}$ of the point $x=\left(x_{1}, \ldots, x_{n}\right)$, including a part of hyperplane $x_{n}=0$ and with smooth boundary $\partial G \in C^{2}, G^{+}=G \cap\left\{x_{n}>0\right\}$,
$G^{-}=G \cap\left\{x_{n}<0\right\}$. The boundary of $G^{+}$consists of a part of hyperplane $x_{n}=0$ for $x_{n}>0$ and smooth surface $\partial G^{+}$. Analogically, the boundary $G^{-}$consists of a part of hyperplane $x_{n}=0$ for $x_{n}<0$ and smooth surface $\partial G^{-}$. Assume that $D=G \times(-T, T), T>0 ; S=\partial G \times(-T, T)$, where $\Gamma=\partial D$ is a boundary of domain $D$. In the domain $D$ consider the system of equations

$$
\begin{aligned}
L_{1}(u, v)= & k_{1}^{(1)}(t) u_{t t}+k_{2}(x) \Delta_{x} u+\sum_{i=1}^{n} a_{i 1}^{(1)}(x, t) u_{x_{i}} \\
& +\sum_{i=1}^{n} a_{i 2}^{(1)}(x, t) v_{x_{i}}+b_{11}(x, t) u_{t}+b_{12}(x, t) v_{t} \\
& +c_{11}(x, t) u+c_{12}(x, t) v=f_{1}(x, t) \\
L_{2}(u, v)= & k_{1}^{(2)}(t) v_{t t}+\Delta_{x} v+\sum_{i=1}^{n} a_{i 1}^{(2)}(x, t) u_{x_{i}} \\
& +\sum_{i=1}^{n} a_{i 2}^{(2)}(x, t) v_{x_{i}}+b_{21}(x, t) u_{t} \\
& +b_{22}(x, t) v_{t}+c_{21}(x, t) u+c_{22}(x, t) v \\
= & f_{2}(x, t),
\end{aligned}
$$

where the $\Delta_{x}$ is Laplace operator $\Delta_{x}=\partial^{2} / \partial x_{1}^{2}+\cdots+\partial^{2} / \partial x_{n}^{2}$.

Everywhere we will assume that the coefficients of the system of (1) are sufficiently smooth. Moreover, the conditions

$$
\begin{gathered}
t k_{1}^{(i)}(t)>0 \text { for } t \neq 0, t \in(-T, T), i=1,2 \\
x_{n} k_{2}(x)<0 \text { for } x_{n} \neq 0, x=\left(x_{1}, \ldots, x_{n}\right) \in G \in R^{n}
\end{gathered}
$$

are satisfied. As far as is known that quadratic form of the equations of system (1) changes, then this system contains portions degenerating elliptic, degenerating hyperbolic, mixed, and composite type differential equations at the same time including changing direction time of variable in the domain $D$. Since, couples form such view system equations in first time considered by author.

Assume the following notations:

$$
\begin{aligned}
& \Gamma_{-T}^{+}=\left\{(x, t) \in \Gamma: x_{n}>0, t=-T\right\}, \Gamma_{-T}^{-}=\{(x, t) \in \Gamma: \\
& \left.x_{n}<0, t=-T\right\}, \\
& \Gamma_{T}^{+}=\left\{(x, t) \in \Gamma: x_{n}>0, t=T\right\}, \Gamma_{T}^{-}=\{(x, t) \in \Gamma: \\
& \left.x_{n}<0, t=T\right\}, S^{+}=\partial G^{+} \times[-T, T], \\
& S^{-}=\partial G^{-} \times[-T, T], D^{+}=D \cap\left\{x_{n}<0\right\}, D^{-}= \\
& D \cap\left\{x_{n}<0\right\} .
\end{aligned}
$$

2.1. The Boundary Value Problem (So-Called Problem "E”). Find the solution of system equations (1) in the domain $\bar{D}$, satisfying the conditions

$$
\begin{array}{ll}
\left.u\right|_{s}=0, & \left.u\right|_{\Gamma_{-T}^{+}}=0,\left.\quad u\right|_{\overline{\Gamma_{T}^{-}}}=0 \\
\left.v\right|_{S}=0, & \left.v\right|_{\overline{\Gamma_{-T}^{+}}}=0,\left.\quad v\right|_{\overline{\Gamma_{T}^{-}}}=0 .
\end{array}
$$


Remark 1. In this situation, the $\Gamma_{-T}^{+}, \Gamma_{-T}^{-}, \Gamma_{T}^{+}$, and $\Gamma_{T}^{-}$set are carriers as boundary conditions which depending on the sign $b_{22}(x, t), k_{1}^{(i)}(t), i=1,2, b_{11}(x, t)$ when the $2 b_{22}-k_{1 t}^{(2)}(t) \geq$ $\delta_{1}>0,2 b_{11}-k_{1 t}^{(1)}(t) \geq \delta>0$ conditions must be satisfied everywhere in $D$. Thus indicated boundary value problems for the system of (1) are put in the forms (3) and (4), where some of the parts of boundary $\Gamma=\partial D$ will be exempt from the boundary conditions. Consequently, the use of the term type of problem "E" and setting the boundary conditions (3) and (4) correspond to and are consistent with the approach cited above.

By the symbol $C_{L}$ we denote a class of twice continuously differentiable functions in the closed domain $D$, satisfying the boundary conditions (3) and (4), by $H_{1, L}(D), H_{2, L}(D)$ in Sobolev's space with weighted spaces obtained by the class $C_{L}$ which is closed by the norms

$$
\begin{aligned}
\|u\|_{H_{1, L}(D)}^{2}= & \int_{D}\left(u_{t}^{2}+\left|k_{2}(x)\right| \sum_{i=1}^{n} u_{x_{i}}^{2}+u^{2}\right) d D, \\
\|u\|_{H_{2, L}(D)}^{2}= & \int_{D}\left(u_{t t}^{2}+k_{2}^{2}(x) \sum_{i=1}^{n} u_{x_{i} x_{i}}^{2}+\left|k_{2}(x)\right| \sum_{i=1}^{n} u_{x_{i} t}^{2}\right. \\
& \left.+\left|k_{2}(x)\right| \sum_{i=1}^{n} u_{x_{i}}^{2}+u_{t}^{2}+u^{2}\right) d D,
\end{aligned}
$$

respectively. Since $k_{2}(x) \neq 0$ for $x_{n} \neq 0$, by Sobolev's embedding theorems [23] the functions from the spaces $H_{2, L}(D)$ will satisfy the boundary conditions (3), (4).

Lemma 2. Let the following conditions

(a) $2 b_{11}(x, t)-k_{1 t}^{(1)}(t) \geq \delta>0$ for $t=0, x \in G$,

(b) $2 b_{22}(x, t)-k_{1 t}^{(2)}(t) \geq \delta_{1}>0$ for $t=0, x \in G$,

(c) $\left|a_{i 1}^{(1)}(x, t)\right|^{2} \leq M_{1}\left|k_{2}(x)\right|,\left|a_{i 1}^{(2)}(x, t)\right|^{2} \leq M_{2}\left|k_{2}(x)\right|$, $\sum_{i=1}^{n}\left(a_{i 1}^{(1)}-k_{2 x_{i}}\right)^{2} \leq M_{3}\left|k_{2}(x)\right|$, where $M_{1}, M_{2}, M_{3}$, and $M$ are sufficiently large constants,

(d) $2 c_{22}(x, t)-\sum_{i=1}^{n} a_{i 2}^{(2)}-b_{22}(x, t) \leq 0,(x, t) \in D$,

(e) $\left|a_{i 2}^{(1)}\right|^{2} \leq M\left|k_{2}(x)\right|$

(f) $-\mu c_{22}-c_{22 t}>0 ; \lambda c_{11}-c_{11 t}>0$ (the constants $\lambda>0$, $\mu>0$ are chosen)

be satisfied. Then there exist constants $\lambda \geq \lambda_{0}>0, \mu \geq \mu_{0}>0$ such that for all functions $u(x, t), v(x, t)$ from the class $c_{L}$ the following inequality holds true:

$$
\begin{aligned}
& \int_{D^{+}} L_{1}(u, v) e^{-\lambda t} u_{t} d D^{+}+\int_{D^{+}} e^{-\lambda t} v_{t} L_{2}(u, v) d D^{+} \\
& \quad+\int_{D^{-}} e^{\mu t} u_{t} L_{1}(u, v) d D^{-}+\int_{D^{-}} e^{\mu t} v_{t} L_{2}(u, v) d D^{-} \\
& \geq m\left(\|u\|_{H_{1}, L^{(D)}}^{2}+\|v\|_{H_{1}, L^{(D)}}^{2}\right)
\end{aligned}
$$

where the constant $m$ is not dependent on function of $u(x, t)$ and $v(x, t)$.
Proof. Let the functions $u(x, t), v(x, t) \in C_{L}$ and consider the following integrals:

$$
\begin{gathered}
J_{1}=\int_{D^{+}} L_{1}(u, v) e^{-\lambda t} u_{t} d D^{+}+\int_{D^{-}} e^{\mu t} u_{t} L_{1}(u, v) d D^{-} \\
J_{2}=\int_{D^{+}} L_{2}(u, v) e^{-\lambda t} v_{t} d D^{+}+\int_{D^{-}} L_{2}(u, v) e^{\mu t} v_{t} d D^{-} .
\end{gathered}
$$

After integration by parts and allowing for boundary conditions of (3), (4) and taking into account nonnegative boundary integrals we get

$$
\left.\begin{array}{rl}
J_{1}= & \int_{D^{+}} L_{1}(u, v) e^{-\lambda t} u_{t} d D^{+}+\int_{D^{-}} e^{\mu t} u_{t} L_{1}(u, v) d D^{-} \\
\geq & \frac{1}{2} \int_{D^{+}}\left\{e ^ { - \lambda t } \left[\left(2 b_{11}-k_{1 t}^{(1)}(t)+\lambda k_{1}^{(1)}(t)\right) u_{t}^{2}\right.\right.
\end{array}\right\}
$$




$$
\begin{gathered}
+2 \sum_{i=1}^{n}\left(a_{i 1}^{(2)} u_{x_{i}} v_{t}+a_{i 2}^{(2)} v_{x_{i}} v_{t}\right) \\
\left.\left.+2 c_{21} u v_{t}+2 b_{21} u_{t} v_{t}\right]\right\} d D^{+} \\
+\int_{D^{-}}\left\{e ^ { \mu t } \left[\left(2 b_{22}-k_{1}^{(2)}(t)-\mu k_{1}^{(2)}(t)\right) v_{t}^{2}\right.\right. \\
+\sum_{i=1}^{n}\left(a_{i 1}^{(2)} u_{x_{i}} v_{t}+a_{i 2}^{(2)} v_{x_{i}} v_{t}\right) \\
+\mu \sum_{i=1}^{n} v_{x_{i}}^{2}+2 c_{21} u v_{t} \\
\left.\left.+2 b_{21} u_{t} v_{t}+\left(-\mu c_{22}-c_{22 t}\right) v^{2}\right]\right\} d D^{-} \\
+\int_{G^{+}} k_{1}^{(2)}(T) e^{-\lambda T} v_{t}^{2}(x, T) d x \\
-\int_{G^{-}} k_{1}^{(2)}(-T) e^{\mu T t} v_{t}^{2}(x,-T) d x \\
-\int_{G^{-}} c_{22}(x,-T) e^{-\mu T} v^{2}(x,-T) d x \\
+\int_{G^{+}} c_{22}(x, T) e^{-\lambda T} v^{2}(x, T) d x .
\end{gathered}
$$

Now, using inequality of Cauchy-Bunyakovskiy, inequality of Poincare, and conditions of Lemma 2 for coefficients of system equations (1) and taking into account chosen constants $\lambda=\lambda_{0}>0, \mu=\mu_{0}>0$ with the fact that the coefficients $k_{1}^{(i)}(t), i=1,2$, are homogeneous on the boundaries and after summarizing estimates for $J_{1}$ and $J_{2}$, then obtain validity of inequality (6).

Definition 3. One says that $u(x, t)$ and $v(x, t)$ are regular solution of problem ((1)-(4)), if the functions $u(x, t), v(x, t) \in$ $H_{2, L}(D)$ satisfy equation of (1) almost everywhere in domain D.

Note that for solvability of problem ((1)-(4)) the standard methods $([3,5-9,13,15-19,24]$, etc.) are not applicable, because the condition of Somigliana [25] is not fulfilled. We need to seek other structure steps of proof or nonclassical method for solvability of problem ((1)-(4)). For this reason, first of all, begin to formulate the theory of existence; first take the decaying system equations in the following form:

$$
\begin{aligned}
L_{1}(u) & =k_{1}^{(1)}(t) u_{t t}+k_{2}(x) \Delta u+\sum_{i=1}^{n} a_{i 1}^{(1)} u_{x_{i}}+b_{11} u_{t}+c_{11} u \\
& =f_{1}(x, t), \\
L_{2}(v) & =k_{1}^{(2)}(t) v_{t t}+\Delta v+\sum_{i=1}^{n} a_{i 2}^{(2)} v_{x_{i}}+b_{22} v_{t}+c_{22} v \\
& =f_{2}(x, t) .
\end{aligned}
$$

For proving solvability of problem ((9), (3)) we use the method of " $\varepsilon$-regularization" and it is the fact that the hyperplane $x_{n}=0$ is characteristic for (9). Therefore, we can consider the boundary value problem ((9), (3)) in the following form.

2.2. Boundary Value Problem 1. Find the solution of (9) in the domain $D^{+}$, satisfying the boundary conditions

$$
\left.u\right|_{\Gamma_{-T}^{+}}=0,\left.\quad u\right|_{s^{+}}=0 .
$$

2.3. Boundary Value Problem 2. Find the solution of (9) in the domain $D^{-}$, satisfying the boundary conditions

$$
\left.u\right|_{\overline{\Gamma_{T}^{-}}}=0,\left.\quad u\right|_{s^{-}}=0 .
$$

By $C_{L}^{\prime}\left(D^{+}\right), C_{L}^{\prime}\left(D^{-}\right)$we denote a class of infinitely differentiable functions in the closed domains $D^{+}, D^{-}$satisfying the boundary conditions (11) and (12), respectively.

\section{Uniqueness Solution of Problem ((1)-(4)) in Space $H_{2, L}(D)$}

Theorem 4. Let the conditions of Lemma 2 be fulfilled. Then the regular solution of the problem ((1)-(4)) is unique.

Proof. Indeed, let $u_{1}, v_{1}$ and $u_{2}, v_{2}$ be two solutions of problem ((1)-(4)) which is satisfying the systems equations (1). Let $u=u_{1}-u_{2}, v=v_{1}-v_{2}$. Then the functions $u, v$ will be satisfying equations $L_{1}(u, v)=0$ and $L_{2}(u, v)=0$ in the domain $D$. Suppose that $u \neq 0, v \neq 0$ are satisfied. Let us take sequence, functions $\left\{u_{n}\right\},\left\{v_{n}\right\} \in C_{L}, n=1,2, \ldots$, and so forth, such that $u_{n} \rightarrow u$ in $H_{2, L}(D)$ for $n \rightarrow \infty, v_{n} \rightarrow v$ in $H_{2, L}(D)$ for $n \rightarrow \infty$. By the inequality of (6) we have

$$
\begin{aligned}
& \left\|L_{1}(u, v)\right\|_{L_{2}(D)}+\left\|L_{2}(u, v)\right\|_{L_{2}(D)} \\
& \geq m_{1}\left(\|u\|_{H_{2, L}(D)}+\|v\|_{H_{2, L}(D)}\right),
\end{aligned}
$$

where the constant $m_{1}$ is independent of the functions $u(x, t)$ and $v(x, t)$. Therefore we can assert that $L_{1}\left(u_{n}, v_{n}\right) \rightarrow$ $L_{1}(u, v), L_{2}\left(u_{n}, v_{n}\right) \rightarrow L_{2}(u, v)$ for $n \rightarrow \infty$. By the virtue of inequality of (6) we have

$$
\begin{aligned}
\left(L_{1}\left(u_{n}, v_{n}\right), e^{-\lambda t} u_{n t}\right)_{L_{2}\left(D^{+}\right)}+\left(L_{1}\left(u_{n}, v_{n}\right), e^{\mu t} u_{n t}\right)_{L_{2}\left(D^{-}\right)} \\
\quad+\left(L_{2}\left(u_{n}, v_{n}\right), e^{-\lambda t} v_{n t}\right)_{L_{2}\left(D^{+}\right)} \\
\quad+\left(L_{2}\left(u_{n}, v_{n}\right), e^{\mu t} v_{n t}\right)_{L_{2}\left(D^{-}\right)} \\
\geq m_{1}\left(\left\|u_{n}\right\|_{H_{1, L}(D)}^{2}+\left\|v_{n}\right\|_{H_{1, L}(D)}^{2}\right) .
\end{aligned}
$$

Hence, passing to limit as $n \rightarrow \infty$ in last inequality, we get $u_{n} \rightarrow 0, v_{n} \rightarrow 0$ in space $H_{1, L}(D)$. On the other sides we have

$$
\begin{gathered}
\left\|u_{n}-u\right\|_{H_{1, L}(D)} \leq\left\|u_{n}-u\right\|_{H_{2, L}(D)} \longrightarrow 0, \\
\left\|v_{n}-v\right\|_{H_{1, L}(D)} \leq\left\|v_{n}-v\right\|_{H_{2, L}(D)} \longrightarrow 0
\end{gathered}
$$


for $n \rightarrow \infty$. Hence, $u \equiv 0, v \equiv 0$. That is proof of Theorem 4 . Now, we need the proof of solvability problem ((1)-(4)).

\section{The Existence of Weak (Regular) Solution of Problems ((9), (11)) and ((9), (12))}

Lemma 5. Let the conditions (a)-(c), (e), and (f) of Lemma 2 be satisfied. Then for any functions $u(x, t) \in C_{L}^{\prime}\left(D^{+}\right)(u(x, t) \in$ $\left.C_{L}^{\prime}\left(D^{-}\right)\right)$the following inequalities

$$
\begin{gathered}
\left(L_{1}(u), e^{-\lambda t} u_{t}\right)_{L_{2}\left(D^{+}\right)} \geq m_{1}\|u\|_{H_{1, L}\left(D^{+}\right)}^{2}, \\
\left(L_{1}(u), e^{\mu t} u_{t}\right)_{L_{2}\left(D^{-}\right)} \geq m_{2}\|u\|_{H_{1, L}\left(D^{-}\right)}^{2}
\end{gathered}
$$

are valid.

Proof. Let us consider the integrals

$$
\begin{aligned}
\int_{D^{+}} L_{1}(u) e^{-\lambda t} u_{t} d D^{+} & =\int_{D^{+}} f_{1} e^{-\lambda t} u_{t} d D^{+} \\
\int_{D^{-}} L_{1}(u) e^{\mu t} u_{t} d D^{-} & =\int_{D^{-}} f_{1} e^{\mu t} u_{t} d D^{-}
\end{aligned}
$$

After integration by parts, allowing for boundary conditions and taking into account nonnegative boundary integrals, we get

$$
\begin{aligned}
& \left(L_{1}(u), e^{-\lambda t} u_{t}\right)_{L_{2}\left(D^{+}\right)} \\
& \geq \frac{1}{2} \int_{D^{+}}\left\{e ^ { - \lambda t } \left[\left(2 b_{11}(x, t)-k_{1 t}^{(1)}(t)+\lambda k_{1}^{(1)}(t)\right) u_{t}^{2}\right.\right. \\
& +2 \sum_{i=1}^{n} a_{i 1}^{(1)} u_{x_{i}} u_{t}+\left(\lambda c_{11}-c_{11 t}\right) u^{2} \\
& \left.\left.+\left(-\lambda \sum_{i=1}^{n} k_{2}(x) u_{x_{i}}^{2}\right)\right]\right\} d D^{+} \\
& +\int_{G^{+}} c_{11}(x, T) e^{\lambda T} u^{2}(x, T) d x \\
& +\int_{G^{+}} k_{1}^{(2)}(T) e^{-\lambda T} u_{t}^{2}(x, T) d x, \quad \forall u(x, t) \in c_{L}^{\prime}\left(D^{+}\right), \\
& \left.(L u), e^{\mu t} u_{t}\right)_{L_{2}\left(D^{-}\right)} \\
& +\frac{1}{2} \int_{D^{-}}\left\{\sum _ { i = 1 } ^ { \mu t } \left[\left(2 b_{11}(x, t)-k_{1 t}^{(1)}(t)-\mu k_{1}^{(1)}(t)\right) u_{t}^{2}\right.\right. \\
& +2 \sum_{i=1}^{n} a_{i 1}^{(1)} u_{x_{i}} u_{t}+\left(\mu c_{11}-c_{11 t}\right) u^{2}
\end{aligned}
$$

$$
\begin{aligned}
& -\int_{G^{-}} c_{11}(x,-T) e^{-\mu T} u^{2}(x,-T) d x \\
& -\int_{G^{-}} k_{1}^{(2)}(-T) e^{\mu T} u_{t}^{2}(x,-T) d x, \quad \forall u(x, t) \in c_{L}^{\prime}\left(D^{-}\right) .
\end{aligned}
$$

Hence, using Cauchy-Bunyakovskiy and Poincare inequalities and taking into account conditions (a)-(c), (e), and (f) of Lemma 2 and chosen constants $\lambda=\lambda_{0}>0, \mu=\mu_{0}>0$ with the fact that the coefficients $k_{1}^{(1)}(t), k_{1}^{(21)}(t)$ are homogeneous on the boundaries, then we get the truth of inequalities (16). Moreover, using Holder's inequality we have

$$
\left\|f_{1}\right\|_{L_{2}\left(D^{+}\right)} \geq m_{1}\|u\|_{H_{1, L}\left(D^{+}\right)}^{2}, \quad\left\|f_{1}\right\|_{L_{2}\left(D^{-}\right)} \geq m_{2}\|u\|_{H_{1, L}\left(D^{-}\right)}^{2},
$$

where the constants $m_{1}$ and $m_{2}$ are independent of the function $u(x, t)$.

Definition 6. The function $u(x, t) \in H_{2, L}\left(D^{+}\right)(u(x, t) \in$ $\left.H_{2, L}\left(D^{-}\right)\right)$is said to be regular solution of problems $((9),(11))$, and $((9),(12))$ if it is generalized solution and satisfies almost everywhere (9) in domain $D^{+}\left(D^{-}\right)$.

Lemma 7. Let the conditions of Lemma 5 be fulfilled. Then regular solution of problems ((9), (11)), and ((9), (12)) is unique.

Proof. Lemma 7 is proved by a way similar to Lemmas 2 and 5. Since the equation of (9) is also degenerating then due to regularizing effect for (9).

In the domain $D^{+}$, we have " $\varepsilon$-regularized" equation of mixed type

$$
\begin{aligned}
L_{1 \varepsilon}\left(u_{\varepsilon}\right)= & k_{1}^{(1)}(t) u_{\varepsilon t t}+\left(k_{2}-\varepsilon\right) \Delta u_{\varepsilon}+b_{11} u_{\varepsilon t} \\
& +\sum_{i=1}^{n} a_{i 1}^{(1)} u_{\varepsilon x_{i}}+c_{11} u_{\varepsilon}=f_{1}(x, t)
\end{aligned}
$$

and we state for it the boundary value problem

$$
\left.u_{\varepsilon}\right|_{x_{n}=0}=0,\left.\quad u_{\varepsilon}\right|_{S^{+}}=0,\left.\quad u_{\varepsilon}\right|_{\Gamma_{-T}^{+}}=0 .
$$

Analogically, we will consider the following boundary value problem:

$$
\begin{gathered}
L_{1 \varepsilon}\left(u_{\varepsilon}\right)=k_{1}^{(1)}(t) u_{\varepsilon t t}+\left(k_{2}+\varepsilon\right) \Delta u_{\varepsilon}+b_{11} u_{\varepsilon t} \\
+\sum_{i=1}^{n} a_{i 1}^{(1)} u_{\varepsilon x_{i}}+c_{11} u_{\varepsilon}=f_{1}(x, t) \\
\left.u_{\varepsilon}\right|_{x_{n}=0}=0,\left.\quad u_{\varepsilon}\right|_{S^{-}}=0,\left.\quad u_{\varepsilon}\right|_{\Gamma_{T}^{-}}=0 .
\end{gathered}
$$

Proceeding from the results of [12] we can affirm the following Remark.

Remark 8. If the conditions of Lemmas 5 and 7 and $2 b_{11}(x, t)-$ $\left|k_{1 t}^{(1)}\right| \geq \delta>0(x, t) \in D$ are satisfied, then for any right-hand side $f_{1}(x, t), f_{1 t}(x, t) \in L_{2}\left(D^{+}\right)\left(f_{1}(x, t), f_{1 t}(x, t) \in L_{2}\left(D^{-}\right)\right)$ 
there exists a unique solution of boundary value problems (20), (21), ((22), (23)) from the space $W_{2}^{2}\left(D^{+}\right)\left(W_{2}^{2}\left(D^{-}\right)\right)$and this solution allows following estimates

$$
\begin{aligned}
& \left\|f_{1}\right\|_{L_{2}\left(D^{+}\right)}^{2}+\left\|f_{1 t}\right\|_{L_{2}\left(D^{+}\right)}^{2} \geq m_{3}\left\|u_{\varepsilon}\right\|_{W_{2}^{2}\left(D^{+}\right)}^{2}, \\
& \left\|f_{1}\right\|_{L_{2}\left(D^{-}\right)}^{2}+\left\|f_{1 t}\right\|_{L_{2}\left(D^{-}\right)}^{2} \geq m_{4}\left\|u_{\varepsilon}\right\|_{W_{2}^{2}\left(D^{-}\right)}^{2},
\end{aligned}
$$

where the constants $m_{3}$ and $m_{4}$ are independent of the function $u(x, t)$.

Proof. This Remark can be proves similarly to Lemmas 2 and 5 and Theorem 4 .

Theorem 9 (on the solvability of problem ((9), (11))). Let the conditions of Lemma 5 and $\left|k_{2 x_{i}} k_{2 x_{j}}\right| \leq M_{1}\left|k_{2}(x)\right|$, $f_{1}(x, t), f_{1 t}(x, t) \in L_{2}\left(D^{+}\right)$, and $2 b_{11}(x, t)-\left|k_{1 t}^{(1)}(t)\right| \geq \delta>$ 0 , for $(x, t) \in D^{+}, i, j=1,2, \ldots, n$, be satisfied. Then there exists a unique regular solution of problem ((9), (11)) from the space $H_{2, L}\left(D^{+}\right)$.

Theorem 10 (on the solvability of problem ((9), (12))). Let the conditions of Lemma 2 and $\left|k_{2 x_{i}} k_{2 x_{j}}\right| \leq M_{1}\left|k_{2}(x)\right|, f_{1}(x, t)$, $f_{1 t}(x, t) \in L_{2}\left(D^{-}\right), 2 b_{11}(x, t)-\left|k_{1 t}^{(1)}(t)\right| \geq \delta>0$, and $f(x, t)$ $\in D^{-}, i, j=1,2, \ldots, n$, be satisfied. Then there exists a unique regular solution of problem ((9), (12)) from the space $H_{2, L}\left(D^{-}\right)$.

Proof of Theorems 9 and 10. The following a priori estimates

$$
\begin{aligned}
& \left\|f_{1}\right\|_{L_{2}\left(D^{+}\right)} \geq m_{5} \int_{D^{+}}\left(u_{\varepsilon t}^{2}+\left|k_{2}-\varepsilon\right| \sum_{i=1}^{n} u_{\varepsilon x_{i}}^{2}+u_{\varepsilon}^{2}\right) d D^{+} \\
& \left\|f_{1}\right\|_{L_{2}\left(D^{-}\right)} \geq m_{6} \int_{D^{-}}\left(u_{\varepsilon t}^{2}+\left|k_{2}+\varepsilon\right| \sum_{i=1}^{n} u_{\varepsilon x_{i}}^{2}+u_{\varepsilon}^{2}\right) d D^{-}
\end{aligned}
$$

hold for the functions $u_{\varepsilon}(x, t) \in W_{2}^{2}\left(D^{+}\right)\left(u_{\varepsilon}(x, t) \in\right.$ $\left.W_{2}^{2}\left(D^{-}\right)\right)$and being the solution of boundary value problems $((20),(21))$, and $((22),(23))$, respectively, where the constants $m_{5}$ and $m_{6}$ are independent of $\varepsilon$ and $u(x, t)$. The proof of these statements is easily obtained by integration by parts and using the Cauchy inequality. Further for obtaining the second a priori estimation we take the function $\xi_{1}(t)$

$$
\xi_{1}(t)= \begin{cases}\equiv 1 & \text { for } t \in(-T,-\eta), \frac{T}{2}>\eta>0, \\ \leq 1 & \text { for } t \in\left[-\eta,-\frac{\eta}{2}\right), \\ \equiv 0 & \text { for } t \in\left[-\frac{\eta}{2}, T\right) .\end{cases}
$$

Then, we consider the function $w_{\varepsilon}(x, t)=\xi_{1}(t) u_{\varepsilon}(x, t)$. Obviously, the function $w_{\varepsilon}(x, t)$ will satisfy the equation

$$
L_{1 \varepsilon} w_{\varepsilon}=\xi_{1} f_{1}+2 k_{1}^{(1)}(t) \xi_{1}^{\prime}(t) u_{\varepsilon t}+k_{1}^{(1)}(t) \xi_{1}^{\prime \prime}(t) u_{\varepsilon}=F_{\varepsilon} .
$$

Hence, by virtue of inequalities (24) and (25), the set of functions $\left\{F_{\varepsilon}(x, t)\right\}$ is uniformly bounded in space $L_{2}\left(D^{+}\right)$. On the other side, in domain

$$
D_{\eta / 2}^{+}=\left\{x \in D,-T<t<-\frac{\eta}{2}\right\}
$$

the equation $L_{1 \varepsilon} w_{\varepsilon}=F_{\varepsilon}$ belongs to elliptical type of equation; therefore, multiplying equation of (27) by $-w_{\varepsilon t t}$ and after integrating by parts in the domain $D^{+}$, allowing boundary conditions, use the Cauchy-Bunyakovskiy inequality we get

$$
\begin{gathered}
\left\|F_{\varepsilon}\right\|_{L_{2}\left(D^{+}\right)} \geq m_{7} \int_{D^{+}}\left(w_{\varepsilon t t}^{2}+w_{\varepsilon}^{2}+w_{\varepsilon t}^{2}+\left|k_{2}-\varepsilon\right| \sum_{i=1}^{n} w_{\varepsilon x_{i}}^{2}\right. \\
\left.+\left|k_{2}-\varepsilon\right| \sum_{i=1}^{n} w_{\varepsilon x_{i} x_{i}}^{2}\right) d D^{+}
\end{gathered}
$$

where constant $m_{7}$ is independent of $\varepsilon, u(x, t)$. Now, let us consider the function $\xi_{2}(t) \in C^{\infty}(-T, T)$ such that $\xi_{2}(t) \equiv 0$ for $-T<t<-2 \eta, \xi_{2}(t) \equiv 1,-\eta<t<T$. Since $0 \leq \xi_{2}(t) \leq 1$ and $\xi_{1}(t)+\xi_{2}(t) \equiv 1$, then $\varphi_{\varepsilon}(x, t)=\xi_{2}(t) u_{\varepsilon}(x, t)$. It is easy to see that the functions $\varphi_{\varepsilon}(x, t)$ satisfy the equation

$$
\begin{aligned}
L_{1 \varepsilon} \varphi_{\varepsilon}= & \xi_{2}(t) f_{1}(x, t)+2 k_{1 t}^{(1)}(t) \xi_{2}^{\prime}(t) u_{\varepsilon}(x, t) \\
& +k_{1}^{(1)}(t) \xi_{2}^{\prime \prime}(t) u_{\varepsilon}=\Phi_{\varepsilon}(x, t) .
\end{aligned}
$$

Hence include the fact that the functions $\Phi_{\varepsilon}(x, t), \Phi_{\varepsilon t}(x, t)$ are uniformly bounded with respect to $\varepsilon$ in the space $L_{2}\left(D^{+}\right)$. Therefore, we can take finite difference

$$
\varphi_{\varepsilon h}=\frac{\varphi_{\varepsilon}(x, t+h)-\varphi_{\varepsilon}(x, t)}{h} .
$$

It is easy to see that the functions $\varphi_{\varepsilon}(x, t)$ satisfy the equation

$$
\begin{aligned}
L_{1 \varepsilon}\left(\varphi_{\varepsilon h}\right) & =\xi_{2}(t) f_{1}+2 k_{1}^{(1)}(t) \xi_{2}^{\prime}(t) u_{\varepsilon t}+k_{1}^{(1)}(t) \xi_{2}^{\prime \prime}(t) u_{\varepsilon} \\
& =\Phi_{\varepsilon h}(x, t) .
\end{aligned}
$$

Using the results on smoothness of the solution of problem ((20), (21)) and a priori estimates (25), (29) and passing to limit as $h \rightarrow 0$ in the obtained inequalities

$$
\begin{aligned}
\left\|\phi_{\varepsilon h}\right\|_{L_{2}\left(D^{+}\right)} \geq m_{8} \int_{D^{+}} & \left(\varphi_{\varepsilon h h}^{2}+\varphi_{\varepsilon}^{2}+\varphi_{\varepsilon h}^{2}+\left|k_{2}-\varepsilon\right|\right. \\
& \left.\cdot \sum_{i=1}^{n} \varphi_{\varepsilon x h_{i}}^{2}+\left|k_{2}-\varepsilon\right| \sum_{i=1}^{n} \varphi_{\varepsilon x_{i} x_{i}}^{2}\right) d D^{+}
\end{aligned}
$$

and establishing relation between the functions $f_{1}(x, t)$ and $\Phi_{\varepsilon}(x, t)$ we get

$$
\begin{aligned}
& \left\|f_{1}\right\|_{L_{2}\left(D^{+}\right)}+\left\|f_{1 t}\right\|_{L_{2}\left(D^{+}\right)} \\
& \geq m_{9}\left(\int _ { D ^ { + } } \left(u_{\varepsilon t t}^{2}+\left|k_{2}-\varepsilon\right| \sum_{i=1}^{n} u_{\varepsilon t x_{i}}^{2}\right.\right. \\
& \left.\quad+u_{\varepsilon t}^{2}+\left|k_{2}-\varepsilon\right| \sum_{i=1}^{n} u_{\varepsilon x_{i}}^{2}+u_{\varepsilon}^{2}\right) d D^{+}
\end{aligned}
$$

$$
\left.\forall u_{\varepsilon}(x, t) \in C_{L}^{\prime}\left(D^{+}\right)\right) \text {. }
$$


From the representations of function $\varphi_{\varepsilon}(x, t)$ and from (20) by standard estimation method, we get $\left|k_{2}-\varepsilon\right| \sum_{i=1}^{n} u_{\varepsilon x_{i} x_{i}} \epsilon$ $L_{2}\left(D^{+}\right)$. Consequently, from the uniformly bounded sequences $\left\{u_{\varepsilon}\right\}$ can be chosen such subsequences $\left\{u_{\varepsilon_{k}}\right\}$ which is weakly converges to the function $u(x, t) \in H_{2, L}\left(D^{+}\right)$. Then passing to limit as $\varepsilon_{k} \rightarrow 0, k \rightarrow \infty$ in the identity

$$
\begin{array}{r}
\left(u_{\varepsilon_{k}}, L_{1 \varepsilon_{k}}^{*} w_{\varepsilon}\right)_{L_{2}\left(D^{+}\right)}=\left(f_{1}, w_{\varepsilon}\right)_{L_{2}\left(D^{+}\right)}, \\
\forall w_{\varepsilon}(x, t) \in C_{0}^{\infty}\left(D^{+}\right),
\end{array}
$$

where $L_{1}^{*}$ is an operator conjugated to the operator $L_{1}$.

Hence,

$$
\left(u, L_{1}^{*} w\right)_{L_{2}\left(D^{+}\right)}=\left(f_{1}, w\right)_{L_{2}\left(D^{+}\right)}, \quad \forall w(x, t) \in C_{0}^{\infty}\left(D^{+}\right) .
$$

Consequently, the function $u(x, t) \in H_{2, L}\left(D^{+}\right)$is generalized solution of problem $((9),(11))$ whilst by virtue of conditions $2 b_{11}(x, 0)-k_{1 t}^{(1)}(0) \geq \delta>0, \forall x \in G$ this solution satisfies (9) almost everywhere for $D^{+}$and by Sobolev's embedding theorems [23] the functions from the spaces $H_{2, L}(D)$ will satisfy the boundary conditions (11) almost everywhere.

In a similar way, repeating all the steps carried out for the domain $D^{+}$for $D^{-}$also we can establish that problem $((9),(12))$ has a generalized solution and belongs to the space $H_{2, L}\left(D^{-}\right)$and satisfies (9) almost everywhere for $D^{-}$and boundary conditions (12).

We conclude the following Remark.

Remark 11. Let the following conditions

(a) $2 b_{11}(x, t)-\left|k_{1 t}^{(1)}(t)\right| \geq \delta \geq 0, \forall(x, t) \in D, b_{11}(x, 0)-$ $k_{1 t}^{(1)}(0) \geq \delta>0 \forall x \in G$,

(b) $\left|a_{i 1}^{(1)}(x, t)\right|^{2} \leq M_{1}\left|k_{2}(x)\right|,\left|a_{i 1}^{(2)}(x, t)\right|^{2} \leq M_{2}\left|k_{2}(x)\right|$, $\left|a_{i 2}^{(1)}\right|^{2} \leq M\left|k_{2}(x)\right| \forall(x, t) \in D, \sum_{i=1}^{n}\left(a_{i 1}^{(1)}-k_{2 x_{i}}\right)^{2} \leq$ $M_{3}\left|k_{2}(x)\right|$, where $M_{1}, M_{2}, M_{3}$, and $M$ are sufficiently large constants,

(c) $\lambda c_{11}-c_{11 t}>0$ (the constants $\lambda>0$ are chosen)

be satisfied. Then for $\forall f_{1}(x, t), f_{1 t}(x, t) \in L_{2}(D)$ there exists generalized solution $u(x, t)$ of problems $((9),(11))$ and $((9)$, (12)) and it belongs to the space $H_{2, L}\left(D^{+}\right)\left(H_{2, L}\left(D^{-}\right)\right)$and for $\varepsilon=0$.

\section{The Theorem of Existence and Uniqueness of Strong (Regular) Solution of Problems $((9),(11))$ and $((9),(12))$}

Definition 12 (following [26]). The function $u(x, t) \in$ $H_{1, L}\left(D^{+}\right)\left(u(x, t) \in H_{1, L}\left(D^{-}\right)\right)$is said to be a strong solution of boundary value problems (20), (21), ((22), (23)), if there exists a sequence of functions $\left\{u_{\varepsilon n}\right\} \in C_{L}^{\prime}\left(D^{+}\right)\left(\left\{u_{\varepsilon n}\right\} \in\right.$ $\left.C_{L}^{\prime}\left(D^{-}\right)\right)$such that equality

$$
\lim _{n \rightarrow \infty}\left\|L_{1}\left(u_{n}\right)-f_{1}(x, t)\right\|_{L_{2}\left(D^{+}\right)}=\lim _{n \rightarrow \infty}\left\|u_{n}-u\right\|_{H_{1, L}\left(D^{+}\right)}=0
$$

is fulfilled in the domain $D^{-}$as well if taken instead of the domain $D^{+}$. holds.

The following theorem on the existence of strong solution

Theorem 13. Let the conditions of Lemma 2 and

$$
\begin{gathered}
\left|k_{2 x_{i}} k_{2 x_{j}}\right| \leq M_{1}\left|k_{2}(x)\right|, \quad i, j=1, \ldots, n, \\
2 b_{11}-\left|k_{1}^{(1)}(t)\right| \geq \delta>0, \quad(x, t) \in D
\end{gathered}
$$

be satisfied. Then for any function $f_{1} \in L_{2}\left(D^{+}\right)\left(f_{1} \in L_{2}\left(D^{-}\right)\right)$ there exists a unique strong solution of boundary value problem ((9), (11)) from the space $H_{1, L}\left(D^{+}\right)$(for the problem ((9), (12)) from $\left.H_{1, L}\left(D^{-}\right)\right)$.

Proof. From Theorems 4, 9, and 10 there exists $u^{+}(x, t)$ solution of problem $((9),(11))$ and $u^{-}(x, t)$ solution of problem $((9),(12))$ in the domains $D^{+}$and $D^{-}$, respectively, belonging, respectively, to the spaces $H_{2, L}\left(D^{+}\right)$and $H_{2, L}\left(D^{-}\right)$. Then by the construction of such spaces there exist sequences $\left\{u_{n}\right\} \in$ $C_{L}^{\prime}\left(D^{+}\right)\left(\left\{u_{n}\right\} \in C_{L}^{\prime}\left(D^{-}\right)\right)$such that

$$
\lim _{n \rightarrow \infty}\left\|u_{n}^{+}-u^{+}\right\|_{H_{2, L}\left(D^{+}\right)}=\lim _{n \rightarrow \infty}\left\|u_{n}^{-}-u^{-}\right\|_{H_{2, L}\left(D^{-}\right)}=0 .
$$

From the obvious inequality

$$
\begin{gathered}
\left\|u_{n}^{+}\right\|_{H_{2, L}\left(D^{+}\right)} \geq m\left\|L_{1}\left(u_{n}^{+}\right)\right\|_{L_{2}\left(D^{+}\right)}, \\
\left\|u_{n}^{-}\right\|_{H_{2, L}\left(D^{-}\right)} \geq m\left\|L_{1}\left(u_{n}^{-}\right)\right\|_{L_{2}\left(D^{-}\right)}
\end{gathered}
$$

it follows that $\left\{L_{1}\left(u_{n}^{+}\right)\right\} \rightarrow f_{1}^{+}$in $L_{2}\left(D^{+}\right)$, for $n \rightarrow \infty$. $\left\{L_{1}\left(u_{n}^{-}\right)\right\} \rightarrow f_{1}^{-}$in $L_{2}\left(D^{-}\right)$, for $n \rightarrow \infty$. Thus, suppose that $f_{1 t}^{+} \in L_{2}\left(D^{+}\right), f_{1 t}^{-} \in L_{2}\left(D^{-}\right)$; then regular solutions $u^{+}$and $u^{-}$are strong solution. We are constructing the sequences of functions $f_{1 n}^{+} \in W_{2}^{1}\left(D^{+}\right), f_{1 n}^{-} \in W_{2}^{1}\left(D^{-}\right)$such that $\left\{f_{1 n}^{+}\right\} \rightarrow$ $f_{1}^{+}$in $L_{2}\left(D^{+}\right),\left\{f_{1 n}^{-}\right\} \rightarrow f_{1}^{-}$in $L_{2}\left(D^{-}\right)$, for $n \rightarrow \infty$. Then for the functions $f_{1}^{+}$and $f_{1}^{-}$there exists strong solution problem of ((9), (11)) and ((9), (12)) from the spaces $H_{2, L}\left(D^{+}\right)$and $H_{2, L}\left(D^{-}\right)$, respectively. So, by inequality of Lemma 2 we have

$$
\begin{aligned}
& \left\|f_{1 n}^{+}\right\|_{L_{2}\left(D^{+}\right)} \geq m\left\|u_{n}^{+}\right\|_{H_{1, L}\left(D^{+}\right)}, \\
& \left\|f_{1 n}^{-}\right\|_{L_{2}\left(D^{-}\right)} \geq m\left\|u_{n}^{-}\right\|_{H_{1, L}\left(D^{-}\right)} .
\end{aligned}
$$

Hence, we can include that $u_{n}^{+} \rightarrow u^{+}$in $H_{1, L}\left(D^{+}\right), u_{n}^{-} \rightarrow$ $u^{-}$in $H_{1, L}\left(D^{-}\right)$, for $n \rightarrow \infty$ and these functions are strong solutions of problems ((9), (11)) and ((9), (12)), respectively.

\section{The Solvability of Problem ((9), (3))}

Remark 14 (gluing solutions in the spaces). Let the functions $u^{+} \in H_{i, L}\left(D^{+}\right), u^{-} \in H_{i, L}\left(D^{-}\right), i=1,2$. Then the constructed function

$$
u(x, t)= \begin{cases}u^{+}(x, t), & (x, t) \in D^{+}, \\ u^{-}(x, t), & (x, t) \in D^{-}\end{cases}
$$

will also be from the class $u(x, t) \in H_{i, L}(D), i=1,2$. 
Proof. Consider for $i=1$. Let us take the functions $\left\{u_{n}\right\} \in$ $C^{\infty}(D),\left\{u_{n}\right\} \in C_{L}^{\prime}\left(D^{+}\right)$, and $\left\{u_{n}\right\} \in C_{L}^{\prime}\left(D^{-}\right)$such that $u(x, t) \in$ $L_{2}(D)$ and also $u_{n} \rightarrow u^{+}$in $H_{1, L}\left(D^{+}\right), u_{n} \rightarrow u^{-}$in $H_{1, L}\left(D^{-}\right)$, $n \rightarrow \infty$. Then we can write

$$
\begin{aligned}
\int_{D} \sqrt{\left|k_{2}\right|} u_{n} \varphi_{x_{n}} d D= & \int_{D^{-}} \sqrt{\left|k_{2}\right|} u_{n} \varphi_{x_{n}} d D^{-} \\
& +\int_{D^{+}} \sqrt{\left|k_{2}\right|} u_{n} \varphi_{x_{n}} d D^{+}, \quad \forall \varphi \in C_{0}^{\infty}(D) .
\end{aligned}
$$

Consequently, we have

$$
\begin{aligned}
\int_{D^{+}}\left(\sqrt{\left|k_{2}\right|} u_{n}\right) \varphi_{x_{n}} d D^{+}= & -\int_{D^{+}}\left(\sqrt{\left|k_{2}\right|} u_{n}\right)_{x_{n}} \varphi d D^{+} \\
& -\int_{D^{-}}\left(\sqrt{\left|k_{2}\right|}\right) u_{n x_{n}} \varphi d D^{-}
\end{aligned}
$$

and it is fulfilled in the domain $D^{-}$as well if taken instead of the domain $D^{+}$.

Then from the inequality of $k_{2 x_{n}}^{2} \leq M\left|k_{2}\right|$ we include that the function $\sqrt{\left|k_{2}\right|} u_{n x_{n}}$ uniformly bounded in space $L_{2}(D)$. Hence, it follows that $u \in H_{1, L}(D)$. Analogically, in case of $i=2$ we need to consider the integral $\int_{D} k_{2} u_{n x_{n}} \varphi_{x_{n}} d D$. Consequently, Remark 14 is proved.

Thus, we have the proof of the following theorem according essentially to a combination of the proofs of Theorems 4, 9, and 10 and Lemmas 2, 5, and 7 and Remark 14.

Note. Compare the classical conditions of "gluing solutions" in $[5,7,9,13,16,17]$ which belong to the boundary conditions, but in this case although the conditions of "gluing solutions" are not written together with conditions (3), (4), finally, the gluing solutions determined by (42), of problem ((1)-(4)), belong to the spaces $H_{i, L}(D), i=1,2$, which are proved.

Now, we can prove the main theorem of solvability of problem ((9), (3)).

Theorem 15 (on the solvability of problem ((9), (3))). Let any conditions of Lemmas 2 and 5 and Theorems 4, 9, 10, and 13 be satisfied. Then for any functions $f_{1}, f_{1 t} \in L_{2}(D)$ there exists a unique generalized solution of problem ((9), (3)) from the space $H_{2, L}(D)$.

Proof. Since on the base of Theorems 9, 10, and 13 there exists a unique solution $u^{+}(x, t), u^{-}(x, t)$ of problems $((9)$, (11)) and $((9),(12))$ from the spaces $H_{2, L}\left(D^{+}\right)$and $H_{2, L}\left(D^{-}\right)$, respectively, then function $u(x, t)$ which is constructed by formula (42) will also be from the class $u(x, t) \in H_{2, L}(D)$ and at the same time it is generalized solution of (9); moreover, the functions $u^{+}(x, t)$ and $u^{-}(x, t)$ are strong generalized solution of problem ((9), (3)).

Consequently, it means that the strong and weak solutions of corresponding problems are identity. It follows that problem $((9),(3))$ is solvability. The uniqueness of problem $((9)$, (3)) follows by means of inequality of Lemma 2 . That is proof of Theorem 4. Analogically, the existence of strong solution of problem ((9), (3)) from the space $H_{1, L}(D)$ can be proved.

\section{On the Solvability of Problem ((1)-(4))}

For proving the solvability of problem ((1)-(4)) we use the method of "continuation by parameter." It holds.

Theorem 16 (on the solvability problem of $((10),(4)))$. Let the conditions

$$
2 c_{22}(x, t)-\sum_{i=1}^{n} a_{i 2}^{(2)}(x, t)-b_{22}(x, t) \leq-\delta_{1}<0, \quad(x, t) \in D,
$$

$$
\left|a_{i 1}^{(2)}(x, t)\right|^{2} \leq M_{1}\left|k_{2}(x)\right|
$$

be satisfied. Then for any functions of $f_{2}(x, t) \in L_{2}(D)$ there exists unique solution of problem ((10), (4)) in the space $H_{2, L}(D)$ (in case, instead of condition of (46), if taken smallest of coefficient $\left|a_{i 1}^{(2)}(x, t)\right|$, then there exists unique solution of problem ((10), (4)) in space $\left.W_{2}^{2}(D)\right)$.

Proof. By virtue of condition (45) and $2 b_{22}-\left|k_{1 t}^{(2)}\right| \geq \delta_{1}>0$ $b_{22}(x, 0)-k_{1 t}^{(2)}(0) \geq \delta_{1}>0 \forall x \in G$; the operator

$$
L_{2}(v)=k_{1}^{(2)}(t) v_{t t}+\Delta v+\sum_{i=1}^{n} a_{i 2}^{(2)} v_{x_{i}}+b_{22} v_{t}+c_{22} v
$$

is coercive. Since the coefficient of $k_{1}^{(2)}(t)$ is sign fixed (according to [10]), then there exists unique solution of problem $((10),(4))$ in space $W_{2}^{1}(D)$. If $v(x, t) \in W_{2}^{1}(D)$ then (according to $[23,24])$ any solution of problem $((10),(4))$ will be element of space $W_{2}^{2}(D)$. Analogically, repeating all the steps carried out for the solution $v(x, t) \in H_{2, L}(D)$ we can also establish that problem $((10),(4))$ has generalized solution if condition (45) is satisfied. Therefore Theorem 16 is proved. Now we must prove solvability of problem ((1)-(4)).

$$
\begin{aligned}
& \text { Let } \\
& M \bar{u}=K \bar{u}_{t t}+\sum_{i=1}^{n} A_{i} \bar{u}_{x_{i}}+B \bar{u}_{t}+C \bar{u}, \\
& N \bar{u}=\sum_{i=1}^{n} P_{i} \bar{u}_{x_{i}}+Q \bar{u}_{t}+R \bar{u}, \quad \text { where } \\
& K=\left(\begin{array}{cc}
k_{1}^{(1)} & 0 \\
0 & k_{1}^{(2)}
\end{array}\right), \quad A_{i}=\left(\begin{array}{cc}
a_{i 1}^{(1)} & 0 \\
0 & a_{i 2}^{(2)}
\end{array}\right), \\
& B=\left(\begin{array}{cc}
b_{11} & 0 \\
0 & b_{22}
\end{array}\right), \quad C=\left(\begin{array}{cc}
k_{2} \Delta+c_{11} & 0 \\
0 & \Delta+c_{22}
\end{array}\right), \\
& P_{i}=\left(\begin{array}{cc}
0 & a_{i 2}^{(1)} \\
a_{i 1}^{(2)} & 0
\end{array}\right), \quad Q=\left(\begin{array}{cc}
0 & b_{12} \\
b_{21} & 0
\end{array}\right), \\
& R=\left(\begin{array}{cc}
0 & c_{12} \\
c_{21} & 0
\end{array}\right), \quad \bar{u}=\left(\begin{array}{cc}
u \\
v
\end{array}\right), \quad \bar{f}=\left(\begin{array}{l}
f_{1} \\
f_{2}
\end{array}\right) .
\end{aligned}
$$

Then the system equations (1) can be written in the form

$$
L \bar{u}=M \bar{u}+N \bar{u}=\bar{f} .
$$


Theorem 17. Let the conditions of Lemmas 2, 5, and 7 and Theorems 4, 9, 10, 13, 15, and 16 are satisfies; moreover $f_{1}, f_{2}, f_{1 t} \in L_{2}(D), f_{2}(x,-T)=0,\left|a_{i 2}^{(1)}\right|^{2} \leq M\left|k_{2}(x)\right|$ are fulfilled. Then there exists a unique solution of problem ((1)(4)) in space $H_{2, L}(D)$. In case that $a_{i 2}^{(1)}$ is smallest then there exists a unique solution of problem ((1)-(4)) from the space $H_{2, L}(D) \cap W_{2}^{2}(D)$.

Proof. Multiplying (49), by the vector $\overline{\eta_{1}}=\left(e^{-\lambda t} u_{t},-v\right)$ in domain $D^{+}, \overline{\eta_{2}}=\left(e^{\mu t} u_{t},-v\right)$ in domain $D^{-}$, after integration by parts and using the Cauchy inequality, allowing for boundary condition (by analogical action to Lemmas 2, 5, and 7), we get the following estimates:

$$
\begin{aligned}
\|L \bar{u}\|_{L_{2}(D)} & \geq m\|\bar{u}\|_{H_{1, L}(D)} \quad \text { or } \\
\|L \bar{u}\|_{L_{2}(D)} & \geq m\|\bar{u}\|_{H_{1, L}(D) \cap W_{2}^{1}(D)} .
\end{aligned}
$$

Now, let $H_{t, 0}$ be the space of vector function $\bar{\varphi}=\left(\varphi_{1}, \varphi_{2}\right)$ such that $\varphi_{1}, \varphi_{1 t}, \varphi_{2} \in L_{2}(D)$ and $\varphi_{1}(x,-T)=0$. The norm of space $H_{t, S}$ is defined by $\|\varphi\|_{t, 0}^{2}=\left\|\varphi_{1 t}\right\|_{0}^{2}+\left\|\varphi_{2}\right\|_{0}^{2}$.

From the results of Theorems 15 and 16, follows a prior estimates

$$
\|\bar{u}\|_{H_{2, L}(D)} \leq p\|M \bar{u}\|_{t, 0} \quad \text { or } \quad\|\bar{u}\|_{H_{2, L}(D) \cap W_{2}^{2}(D)} \leq p\|M \bar{u}\|_{t, 0},
$$

where $p$ constant is not dependent on $\bar{u}(x, t)$. We must show that analogical estimates (50) and (51) can be expressed for operator $L u$. Indeed, we may write $M \bar{u}=L \bar{u}-N \bar{u}$; then

$$
\begin{aligned}
& \|\bar{u}\|_{H_{2, L}(D)} \leq p\left(\|L \bar{u}\|_{t, 0}+\|N \bar{u}\|_{t, 0}\right) \quad \text { or } \\
& \|\bar{u}\|_{H_{2, L}(D) \cap W_{2}^{2}(D)} \leq p\left(\|L \bar{u}\|_{t, 0}+\|N \bar{u}\|_{t, 0}\right)
\end{aligned}
$$

is valid. Now, we consider the set of equations $L_{\tau} \bar{u}=M \bar{u}+$ $\tau N \bar{u}$ where $0 \leq \tau \leq 1$. Obviously, the following a prior estimate is uniformly bounded with respect to parameter of $\tau:$

$$
\|\bar{u}\|_{H_{2, L}(D)} \leq c_{1}\left\|L_{\tau} \bar{u}\right\|_{t, 0},
$$

where $c_{1}$ is independent of parameter $\tau$ and $\bar{u}(x, t)$. On the other hand for $\tau=0$ we have $L_{0} \bar{u}=M \bar{u}$. In this case we considered problem is solvable. Notice that if $\tau=1$ then $L_{1}=L$. Then as well as known method of continuation by parameter we can prove solvability of problem ((1)-(4)).

\section{Conclusion}

The existence and uniqueness of the boundary value problem for linear systems equations of the mixed hyperbolicelliptic type in the multivariate domain with the changing time direction are studied. The existence and uniqueness of generalized and regular solutions of a boundary problem are established in a weighted Sobolev space. Moreover, applying the result of the monograph [22], difference schemes for the numerical solution of the boundary value problem for linear systems equations of the mixed hyperbolic-elliptic type in the multivariate domain with the changing time direction can be presented. Of course, the stability estimates for the solution of these difference schemes have been established without any assumptions about the grid steps.

\section{Conflict of Interests}

The author declares that there is no conflict of interests.

\section{Acknowledgments}

The author would like to thank Prof. Allaberen Ashyralyev (Fatih University, Turkey), for his helpful suggestions for the improvement of this paper. The author would like to thank the reviewer who has read the paper very attentively and gave important and essential comments which helped in improvement of this paper.

\section{References}

[1] L. Bers, Mathematical Aspects of Subsonic and Transonic Gas Dynamics, vol. 3 of Surveys in Applied Mathematics, John Wiley \& Sons, New York, NY, USA; Chapman \& Hall, London, UK, 1958.

[2] F. I. Frankl, Selected Works in Gas Dynamics, Nauka, Moscow, Russia, 1973, (Russian).

[3] S. Čanić, B. L. Keyfitz, and E. H. Kim, "Mixed hyperbolic-elliptic systems in self-similar flows," Boletim da Sociedade Brasileira de Matemática, vol. 32, no. 3, pp. 377-399, 2001.

[4] B. Pini, "Un Problem di Valoru ol Contorno Por unequazional a Derivative Puzziali Def Terro Ardine Con Parto Principale di Tipo Composite," Rendiconti del Seminario della Facoltà di Scienze dell'Università di Cagliari, vol. 27, p. 114, 1957.

[5] C. S. Morawetz, "Mixed equations and transonic flow," Journal of Hyperbolic Differential Equations, vol. 1, no. 1, pp. 1-26, 2004.

[6] C. S. Morawetz, "A weak solution for a system of equations of elliptic-hyperbolic type," Communications on Pure and Applied Mathematics, vol. 11, pp. 315-331, 1958.

[7] V. N. Vragov, Boundary Value Problems for the Nonclassical Equations of Mathematical Physics, NSU, Novosibirsk, Russia, 1983, (Russian).

[8] S. G. Pyatkov, "On the solvability one boundary value problem for a forward-backward equation parabolic type," Doklady Akademii Nauk SSSR, no. 6, pp. 1322-1327, 1985.

[9] S. A. Tersenov, "On some problems for forward-backward parabolic equation," Siberian Mathematical Journal, vol. 51, pp. 338-345, 2010

[10] M. A. Nurmamedov, "The first boundary value problems for the equation of mixed type," in Proceedings of the Non-Classical Equations of Mathematical Physics, pp. 117-122, Institute of Mathematics of Siberian Branch of the Academy of Sciences, USSR, Novosibirsk, Russia, 1985 (Russian).

[11] M. A. Nurmamedov, "Some of well-posed boundary value problems for equations of mixed type with orthogonal linear degeneration," in Some Problems of Differential Equations and Discrete Mathematics, pp. 104-107, Novosibirsk State University, Novosibirsk, Russia, 1986, (Russian).

[12] M. A. Nurmamedov, "On the solvability of the first local boundary value problems for linear systems equations of nonclassical type with second order," Journal Reports of Adigeyskiy International Academy of Sciences, vol. 10, no. 2, pp. 51-58, 2008. 
[13] A. V. Bitsadze, Some Classes of Partial Differential Equations, Gordon and Breach, New York, NY, USA, 1988, Russian edition, Nauka, Moscow, Russia, 1981.

[14] G. Fichera, "On a unified theory of boundary value problems for elliptic-parabolic equations of second order," in Boundary Problems in Differential Equations, pp. 97-120, University of Wisconsin Press, Madison, Wis, USA, 1960.

[15] M. V. Keldysh, "On certain cases of degeneration of equations of elliptic type on the boundry of a domain," Doklady Akademii Nauk SSSR, vol. 77, pp. 181-183, 1951 (Russian).

[16] T. H. Otway, The Dirichlet Problem for Elliptic-Hyperbolic Equations of Keldysh Type, vol. 2043 of Lecture Notes in Mathematics, Springer, Berlin, Germany, 2012.

[17] D. Lupo, C. S. Morawetz, and K. R. Payne, "On closed boundary value problems for equations of elliptic-hyperbolic type," Communications on Pure and Applied Mathematics, vol. 60, no. 9, pp. 1319-1348, 2007.

[18] N. Popivanov, "The theory of linear systems of first order pde and equations of mixed type with two perpendicular lines of parabolic degeneracy," in Mathematics and Mathematical Educations: Proceedings of the Second Spring Conference Bulgarian Mathematical Society, pp. 175-181, 1974.

[19] D. Lupo, K. Payne, and N. Popivanov, "Nonexistence of nontrivial solutions for super-critical equations of mixed elliptichyperbolic type," Progress in Nonlinear Differential Equations and Their Applications, vol. 66, pp. 371-390, 2006.

[20] A. Ashyralyev and H. A. Yurtsever, "The stability of difference schemes of second-order of accuracy for hyperbolic-parabolic equations," Computers \& Mathematics with Applications, vol. 52, no. 3-4, pp. 259-268, 2006.

[21] A. Ashyralyev and Y. Ozdemir, "On stable implicit difference scheme for hyperbolic-parabolic equations in a Hilbert space," Numerical Methods for Partial Differential Equations, vol. 25, no. 5, pp. 1100-1118, 2009.

[22] A. Ashyralyev and P. E. Sobolevskii, New Difference Schemes for Partial Differential Equations, vol. 148 of Operator Theory Advances and Applications, Birkhäuser, Basel, Switzerland, 2004.

[23] S. L. Sobolev, Applications of Functional Analysis in Mathematical Physics, Izdat. Leningrad. Gos. Univ., Leningrad, Russia, 1950, English transl. Amer. Math. Soc, Providence, RI, USA, 1963.

[24] O. A. Ladyzhenskaya, The Boundary Value Problems of Mathematical Physic, vol. 49 of Applied Mathematical Sciences, Springer, New York, NY, USA, 1985.

[25] C. Somigliana, "Sui sistemi simmetrici di equazioni a derivate parziali," Annali di Matematica Pura ed Applicata, vol. 22, no. 1, pp. 143-156, 1894.

[26] L. Sarason, "On weak and strong solutions of boundary value problems," Communications on Pure and Applied Mathematics, vol. 15, no. 3, pp. 237-288, 1962, MR27 \#460. 


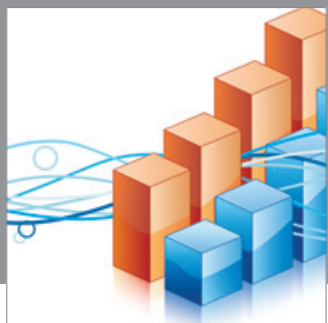

Advances in

Operations Research

mansans

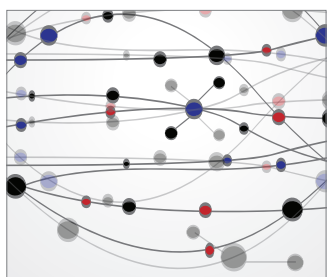

The Scientific World Journal
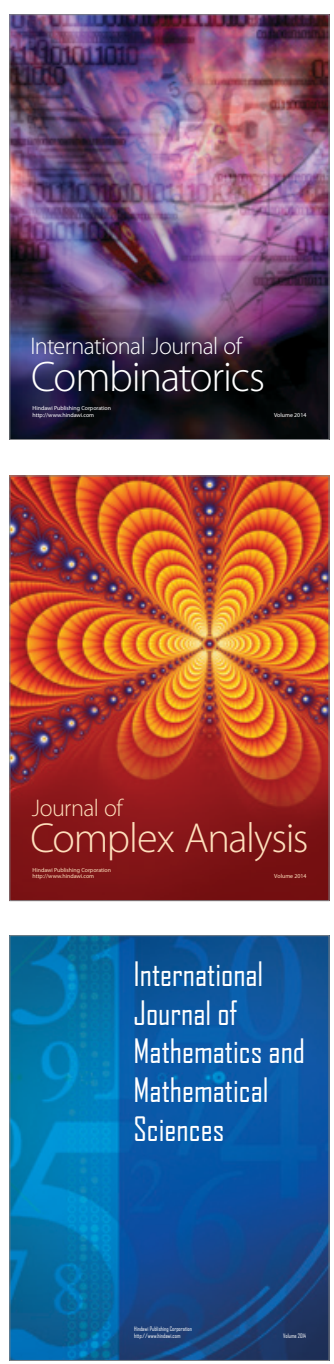
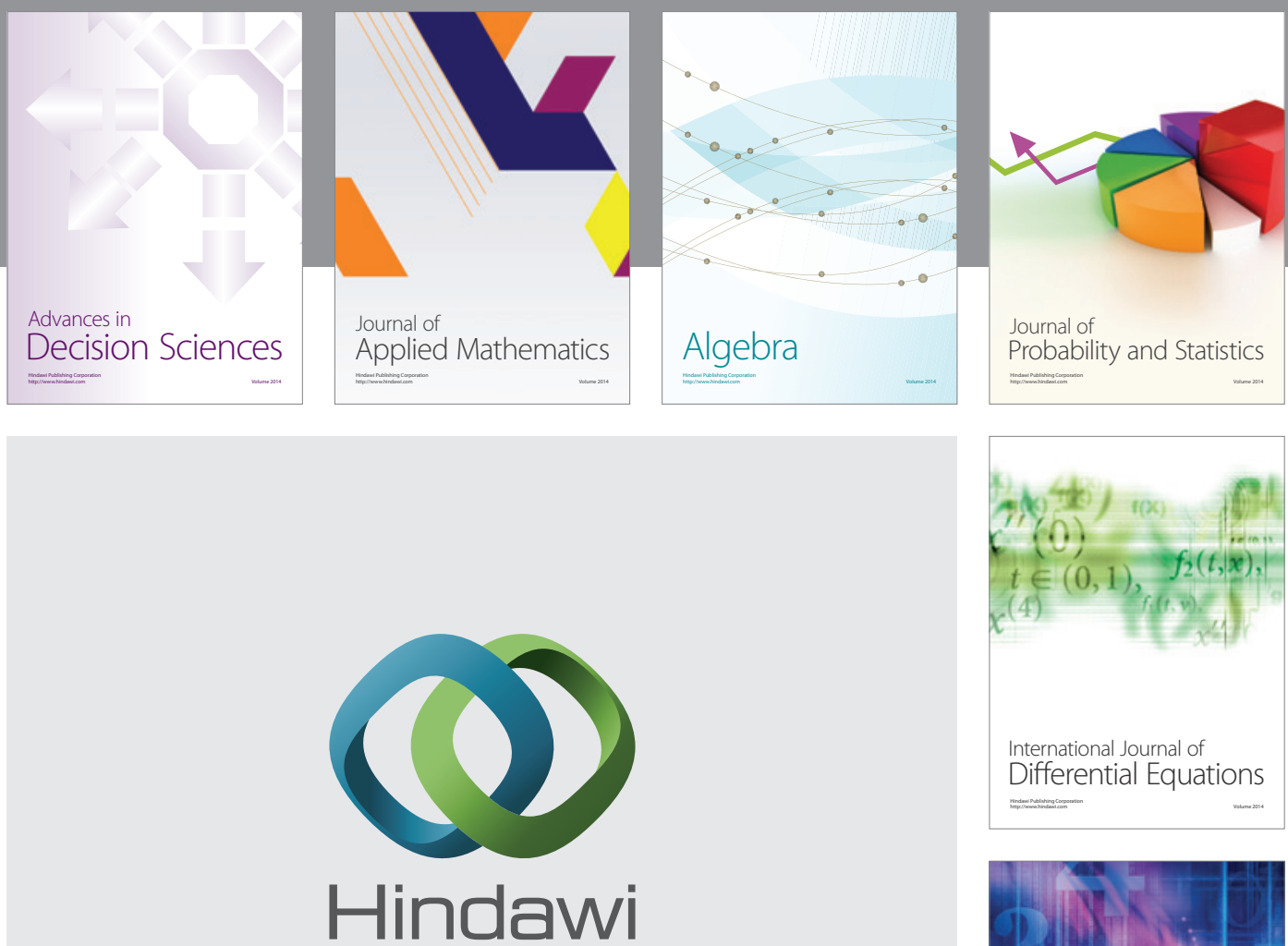

Submit your manuscripts at http://www.hindawi.com
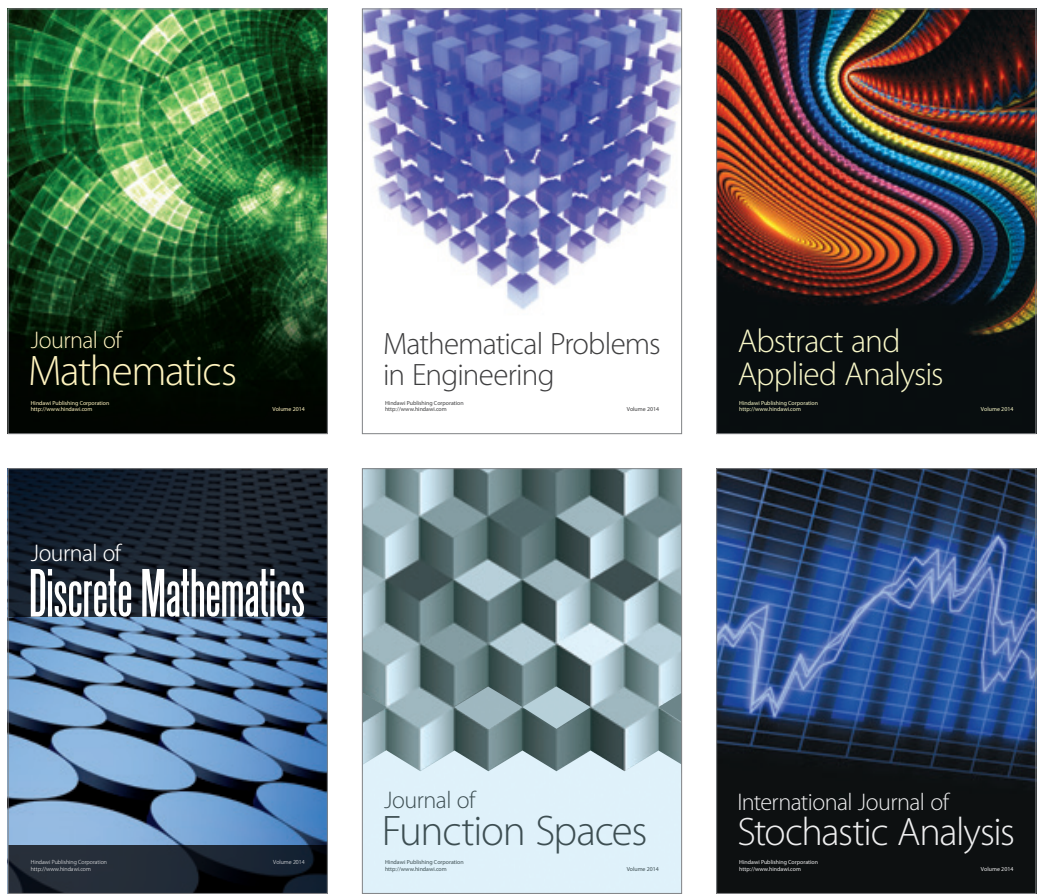

Journal of

Function Spaces

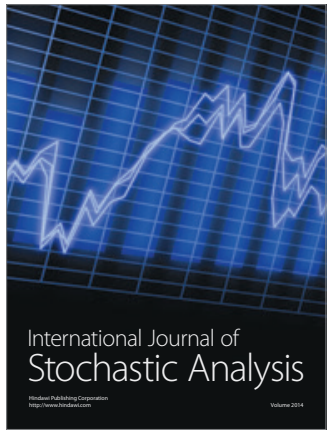

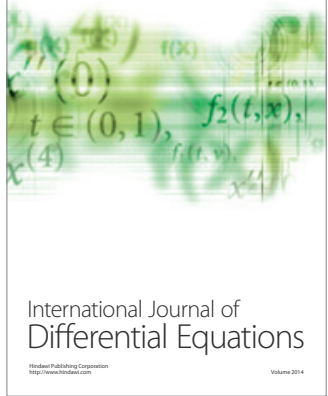
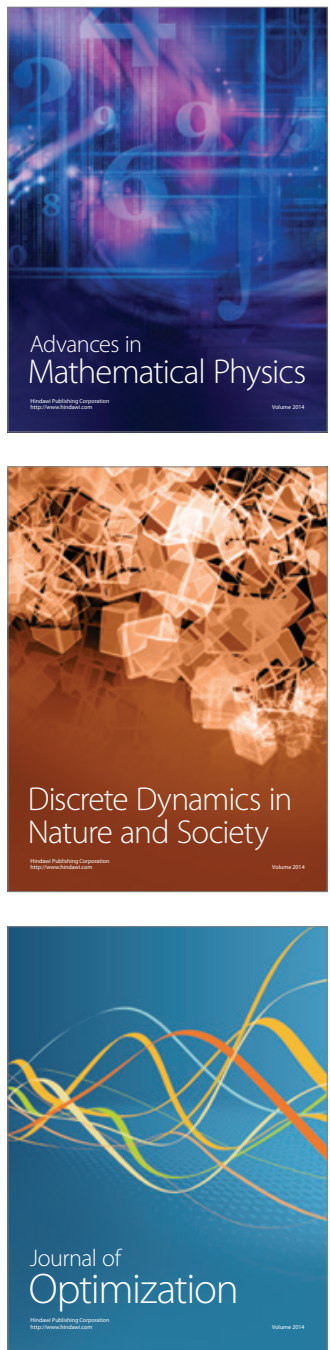